\title{
Evolution of single-particle structure near the $N=20$ island of inversion
}

\author{
P. T. MacGregor, ${ }^{1}$ D. K. Sharp $\odot,{ }^{1,}{ }^{*}$ S. J. Freeman, ${ }^{1}$ C. R. Hoffman, ${ }^{2}$ B. P. Kay, ${ }^{2}$ T. L. Tang, ${ }^{2}$ L. P. Gaffney, ${ }^{3,4}$ E. F. Baader, ${ }^{4}$ \\ M. J. G. Borge, ${ }^{5}$ P. A. Butler, ${ }^{3}$ W. N. Catford, ${ }^{6}$ B. D. Cropper, ${ }^{1}$ G. de Angelis, ${ }^{7}$ J. Konki, ${ }^{4}$ Th. Kröll, ${ }^{8}$ M. Labiche, ${ }^{9}$ \\ I. H. Lazarus, ${ }^{9}$ R. S. Lubna, ${ }^{10,11}$ I. Martel, ${ }^{3,12}$ D. G. McNeel, ${ }^{13}$ R. D. Page, ${ }^{3}$ O. Poleshchuk,,${ }^{14}$ R. Raabe,${ }^{14}$ F. Recchia, ${ }^{15,16}$ \\ and J. Yang ${ }^{14}$ \\ ${ }^{1}$ Department of Physics and Astronomy, University of Manchester, Manchester, M13 9PL, United Kingdom \\ ${ }_{2}^{2}$ Physics Division, Argonne National Laboratory, Argonne, Illinois 60439, USA \\ ${ }^{3}$ Oliver Lodge Laboratory, University of Liverpool, Liverpool L69 7ZE, United Kingdom \\ ${ }^{4}$ CERN, CH-1211 Geneva 23, Switzerland \\ ${ }^{5}$ Instituto de Estructura de la Materia, CSIC, E-28006 Madrid, Spain \\ ${ }^{6}$ Department of Physics, University of Surrey, Guildford GU2 5XH, United Kingdom \\ ${ }^{7}$ INFN, Laboratori Nazionali di Legnaro, 35020 Legnaro, Italy \\ ${ }^{8}$ Institut für Kernphysik, Technische Universität Darmstadt, 64289 Darmstadt, Germany \\ ${ }^{9}$ STFC Daresbury Laboratory, Daresbury, Warrington WA4 4AD, United Kingdom \\ ${ }^{10}$ Department of Physics, Florida State University, Tallahassee, Florida 32306, USA \\ ${ }^{11}$ TRIUMF, Vancouver, British Columbia V6T 2A3, Canada \\ ${ }^{12}$ Universidad de Huelva, 21071 Huelva, Spain \\ ${ }^{13}$ Department of Physics, University of Connecticut, Storrs, Connecticut 06269, USA \\ ${ }^{14}$ KU Leuven, Instituut voor Kern- en Stralingsfysica, 3001 Leuven, Belgium \\ ${ }^{15}$ Dipartimento di Fisica e Astronomia dell' Universitá degli Studi di Padova, Padova 35131, Italy \\ ${ }^{16}$ INFN Sezione di Padova, Padova 35131, Italy
}

(Received 5 May 2021; accepted 5 October 2021; published 8 November 2021)

\begin{abstract}
The single-particle properties of ${ }^{29} \mathrm{Mg}$ have been investigated via a measurement of the ${ }^{28} \mathrm{Mg}(d, p){ }^{29} \mathrm{Mg}$ reaction, in inverse kinematics, using the ISOLDE Solenoidal Spectrometer. The negative-parity intruder states from the $f p$ shell have been identified and used to benchmark modern shell-model calculations. The systematic data on the single-particle centroids along the $N=17$ isotones show good agreement with shell-model predictions in describing the observed trends from stability toward ${ }^{25} \mathrm{O}$. However, there is also evidence that the effect of the finite geometry of the nuclear potential is playing a role on the behavior of the $p$ orbitals near the particle-emission threshold.
\end{abstract}

DOI: 10.1103/PhysRevC.104.L051301

Neutron-rich magnesium isotopes $(Z=12)$ exist in a rapidly evolving region in terms of nuclear structure. Magnesium exhibits the most abrupt transition into the $N=20$ island of inversion, first identified by anomalous groundstate binding energies [1,2]. Intruder configurations, involving excitations across the $N=20$ gap, fall in excitation energy as $N$ increases, leaving ${ }^{30} \mathrm{Mg}$ lying outside and ${ }^{31} \mathrm{Mg}$ inside the region of inversion [3,4]. A weaker shell gap enhances the contribution of intruder configurations until, inside the island, they become the dominant component of the ground state. The $N=20$ shell gap also disappears along $N=16$ as protons are removed; the separation of the $v d_{3 / 2}$ orbital and the $v f p$ shell reduces and a new

\footnotetext{
*Corresponding author: david.sharp@ manchester.ac.uk
}

Published by the American Physical Society under the terms of the Creative Commons Attribution 4.0 International license. Further distribution of this work must maintain attribution to the author(s) and the published article's title, journal citation, and DOI. shell gap opens at $N=16$, leading to a new doubly magic system, ${ }^{24} \mathrm{O}[5]$.

Here we present a study of ${ }^{29} \mathrm{Mg}$ probed in a measurement of the single-neutron-adding ${ }^{28} \mathrm{Mg}(d, p){ }^{29} \mathrm{Mg}$ reaction with a radioactive beam in inverse kinematics. The results reveal the changes in the single-neutron centroids outside $N=16$, when moving from stability toward the neutron drip line at ${ }^{25} \mathrm{O}$. The structure of ${ }^{29} \mathrm{Mg}$, on the border of the island of inversion, provides useful information on single-particle evolution, in particular, the nature of negative-parity intruder states that are important in driving shape transitions in the island of inversion.

Shell-model calculations in this region have often required ad hoc changes to reproduce data. For example, single-particle energies in the WBP interaction [6] needed to be shifted by 1.8 and $0.5 \mathrm{MeV}$ for $v f_{7 / 2}$ and $v p_{3 / 2}$ orbitals, respectively, to better match data in ${ }^{34} \mathrm{P}_{19}$ [7]. Similarly, in ${ }^{30} \mathrm{Al}_{17}$, WBP energies had to be shifted by $\approx 1 \mathrm{MeV}$ to properly describe negative-parity states [8]. Neither the SDPF-M [9] nor WBP interactions could reproduce the energies of the negativeparity states in ${ }^{27} \mathrm{Ne}_{17}$ [10]. The present measurement 
provides information on single-particle properties of negativeparity states in ${ }^{29} \mathrm{Mg}$ that is needed to properly understand cross-shell effective interactions and to provide important benchmarks for developing new interactions.

Energy levels in ${ }^{29} \mathrm{Mg}$ have been obtained through a variety of techniques such as $\beta$ decay (Ref. [11], for example), $\beta$-delayed spectroscopy [12], and multinucleon transfer reactions (Refs. [13-15]). Single-neutron knock-out reactions from ${ }^{30} \mathrm{Mg}$ have been measured [16], but these probe hole states and only weakly populate the negative-parity states of interest here. A measurement of the ${ }^{28} \mathrm{Mg}(d, p){ }^{29} \mathrm{Mg}$ reaction extracted cross sections for the strongest fragments of strength [17]; the resolving power of the technique used here allowed a more comprehensive study of ${ }^{29} \mathrm{Mg}$, in particular, the detailed fragmentation of single-particle states.

Single-nucleon transfer-reactions are an ideal probe for single-particle information. Angular distributions enable the assignment of the orbital angular momentum $\ell$ transferred. Comparisons of experimental cross sections with predictions of reaction models allow the extraction of spectroscopic factors, reduced cross sections dependent on the overlap between initial and final states, that provide a measure of singleparticle content.

Here we used a solenoid magnet to transport light ions, emitted following reaction of the beam with a deuteratedpolyethylene target, to a position-sensitive array $[18,19]$ aligned along the magnetic-field axis. This technique yields excellent $Q$-value resolution without needing additional detectors, such as $\gamma$-ray arrays. It allows studies of long-lived states and states above particle-emission thresholds, which have no prompt $\gamma$ rays. The new ISOLDE Solenoidal Spectrometer (ISS) exploits this technique with radioactive beams produced via the isotope separation online technique.

The HIE-ISOLDE Linac [20] delivered a beam of ${ }^{28} \mathrm{Mg}$ at $9.47 \mathrm{MeV} / u$ with intensities of $\approx 1 \times 10^{6} \mathrm{pps}$. Magnesium atoms were produced following the bombardment of a siliconcarbide target by $1.4-\mathrm{GeV}$ protons and resonantly ionized by the resonance ionization laser ion source (RILIS) [21], mass separated and injected into an ion trap [22], raised to a $9^{+}$charge state using an electron-beam ion source [23], and injected into the linac. Reactions from small amounts of ${ }^{28} \mathrm{Si}$ beam contamination $(<10 \%)$ were identified and separated from the events of interest (see below).

ISS consists of a large-bore superconducting solenoid, operated at $2.5 \mathrm{~T}$ with the magnetic axis along the beam axis. The beam passes through a hollow position-sensitive silicon detector (PSD) array, redeployed from the HELIOS spectrometer [18]. Protons emitted from the $\mathrm{CD}_{2}$ target (also on axis) in the backward hemisphere follow a helical orbit in the field, returning to axis after a cyclotron period, where they are detected in the PSD array. Two array-to-target distances were used $(-137$ and $-107 \mathrm{~mm}$, as measured from the target to the nearest detector edge) giving a center-of-mass angle coverage of $10^{\circ}<\theta_{\text {c.m. }}<35^{\circ}$ for population of most of the final states. Two targets of nominal thicknesses 80 and $120 \mu \mathrm{g} / \mathrm{cm}^{2}$ were used.

An annular silicon detector was positioned $125.7 \mathrm{~mm}$ downstream of the target to monitor elastically scattered deuterons, providing a measurement of the product of tar-

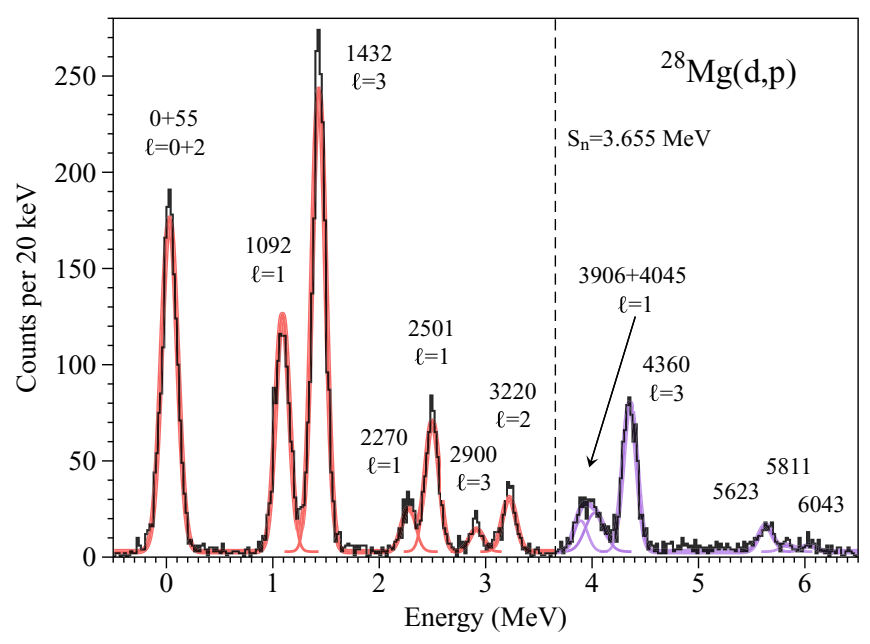

FIG. 1. Excitation spectrum for states populated in the ${ }^{28} \mathrm{Mg}(d, p){ }^{29} \mathrm{Mg}$ reaction at $9.47 \mathrm{MeV} / u$ across all the array. States are labeled with energy in $\mathrm{keV}$ and angular-momentum transfer. The dotted line indicates the neutron-separation energy. Gaussian fits to peaks are overlaid for bound (red) and unbound (purple) states.

get thickness and beam dose. The elastic-scattering cross section, required to determine this product, was calculated using the optical-model parameters described below. Absolute cross sections were extracted with an estimated uncertainty of $\approx 25 \%$, with dominant sources of error arising from uncertainties in detector position and elastic-scattering cross section. A silicon recoil telescope, situated $141 \mathrm{~cm}$ downstream of the target and divided into four quadrants, consisted of $\approx 65 \mu \mathrm{m} \Delta E$ and $\approx 500 \mu \mathrm{m} E$ detectors. This provided particle identification to remove reactions arising from beam contaminants and a timing reference to identify proton-recoil coincidences via time-of-flight measurements. More detail on the setup and detector performance can be found in Ref. [24].

Figure 1 shows the resulting excitation-energy spectrum for states in the residual ${ }^{29} \mathrm{Mg}$ nucleus, after application of recoil energy and coincidence conditions. Unbound states are identified through measurement of ${ }^{28} \mathrm{Mg}$ recoils in coincidence with a proton in the array. The energies of states have been calibrated using known excitation energies from Ref. [25]. An excitation-energy resolution of $\approx 150 \mathrm{keV}$ fullwidth-half-maximum was obtained.

Yields were extracted for nine peaks at up to 12 center-ofmass angles to construct angular distributions. An additional three peaks above $5.5 \mathrm{MeV}$ were identified in a spectrum combining all angles, yielding only an integrated cross section over the $\theta_{\text {c.m. }}$. acceptance of the array. Figure 2 shows the measured angular distributions compared to distorted-wave Born approximation (DWBA) calculations performed using the finite-range code DWUCK5 [26]. Choices of input parameters are consistent with those from the systematic study in Ref. [27]. Calculations for transfer to unbound states used the prescription of Ref. [28]. The $\theta_{\text {c.m. }}$ range of the distributions is determined by the angular acceptance of the on-axis array and recoil detectors. In particular, the latter limits the forward-angle coverage of higher lying states, which can be 


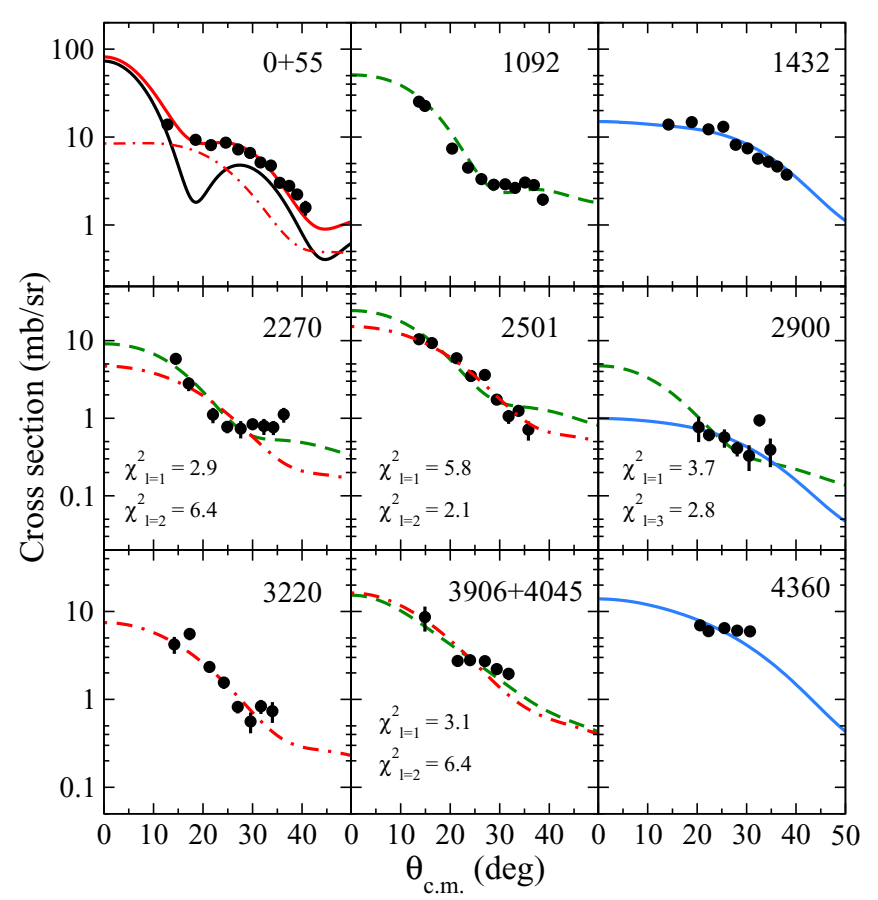

FIG. 2. Angular distributions for states observed in the ${ }^{28} \mathrm{Mg}(d, p){ }^{29} \mathrm{Mg}$ reaction, labeled by energy in $\mathrm{keV}$. The lines are DWBA calculations for angular-momentum transfer with $\ell=0$ (black), 1 (green dashed), 2 (red hatched), and 3 (blue). The solid red line for the ground-state doublet denotes a sum of the two distributions. Where new assignments are made, possible DWBA fits are shown with the value of the reduced $\chi^{2}$.

mitigated using array-singles data for stronger states (see Ref. [24]).

The lowest peak is formed from the ground state and an excited state at $54.60 \mathrm{keV}$ [11] and is confirmed as a doublet with $\ell=2+0$. The strongest populated states are at 1092 and $1432 \mathrm{keV}$, with $\ell=1$ and $\ell=3$, respectively. These are all in agreement with the previously observed states in transfer [17] and knock-out reactions [16]. Newly resolved states at 2270, 2501, 2900, and $3220 \mathrm{keV}$ were observed; these were previously assigned as an $\ell=2+3$ doublet at $2.4 \mathrm{MeV}$ in Ref. [17]. Here, the $2270-\mathrm{keV}$ state has been assigned $\ell=1$ using angular distributions and that at $2900 \mathrm{keV}$ tentatively favours $\ell=3$. The $2501-\mathrm{keV}$ state has previously been populated by knockout from either a $d$ or $f$ orbital, with the angular distribution here confirming an $\ell=2$ assignment. The state at $3220 \mathrm{keV}$, previously observed in knockout as being from a $d$ orbital, is confirmed here. Two peaks above the neutron separation energy $\left(S_{n}=3655(12) \mathrm{keV}\right.$ [29]) at 3980 and $4360 \mathrm{keV}$ have angular distributions tentatively indicating $\ell=1$ and 3 , respectively. The latter has a vanishingly small particle-decay width, favoring $\ell=3$, and was also observed in Ref. [17] with an $\ell=3$ distribution. The $\ell=1$ peak has not previously been observed. While the assignment based on the distribution is tentative, the significant natural width of this peak (fit as either a singlet $\Gamma_{s p} \approx 200 \mathrm{keV}$ or a doublet $\Gamma_{s p} \approx 17-85 \mathrm{keV}$ ) favors an $\ell=1$ assignment; arguments are presented later for a doublet with states at 3906 and $4045 \mathrm{keV}$.

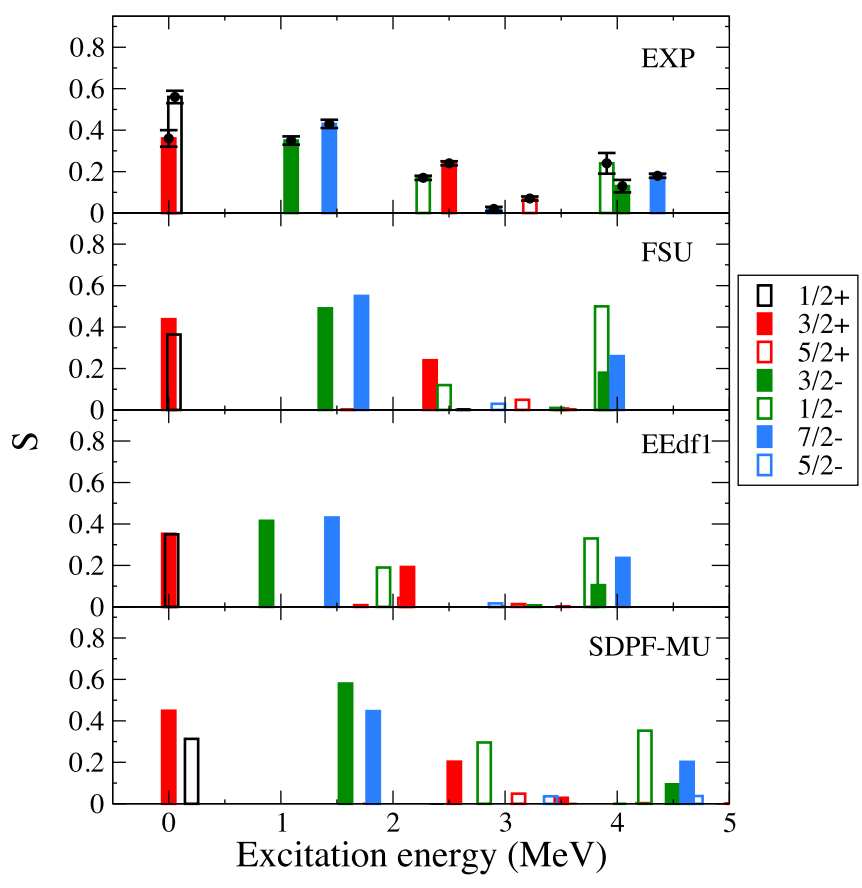

FIG. 3. Spectroscopic factors from the present work (EXP) for states with $J^{\pi}=1 / 2^{+}$(unfilled black), 1/2,3/2- (unfilled/filled green), $3 / 2,5 / 2^{+}$(filled/unfilled red), and 5/2,7/2- (unfilled/filled blue), with statistical errors. Spectroscopic factors from shell-model calculations are given for the interactions described in the text. The states at 3906 and $4045 \mathrm{keV}$ have been shown here as $1 / 2^{-}$and $3 / 2^{-}$ respectively, but the reverse order is equally possible.

Angular-momentum transfer with $\ell>1$ would have much smaller widths. State-by-state information is available as Supplemental Material [30].

Spectroscopic factors were extracted using DWBA calculations, which are known to carry an uncertainty in absolute normalization. Here we follow recent approaches (for example, Ref. [27]) using the Macfarlane-French sum rules [31]. We determine a value for the overall normalization by ensuring the summed spectroscopic strength for $\ell=0$ and $\ell=2$ is equal to the expected vacancy below $N=20$, which is four neutrons for ${ }^{28} \mathrm{Mg}$. This assumes that all fragments lie low enough to be observed, but the shell-model calculations below agree that $>95 \%$ is carried by observed states, with FSU calculations extending to $8 \mathrm{MeV}$.

Comparisons are made below with previous $(d, p)$ crosssection data leading to ${ }^{31} \mathrm{Si}$ [32] and ${ }^{33} \mathrm{~S}[33,34]$ and a consistent methodology was used, employing the same DWBA modeling and normalization method. Some variation in values of the normalization between different data sets was noted. This might suggest an issue of comparability between absolute cross sections but, ultimately, conclusions are drawn from centroids of strength, which only depend on the relative values within each data set.

In Fig. 3, spectroscopic factors are shown on a state-bystate basis and compared to shell-model calculations using the SDPF-MU [35] and the newer FSU [36] and EEdf1 [37] interactions. These use cross-shell $s d p f$ model spaces, but the FSU interaction also included the $0 p$ orbital. The FSU 
TABLE I. Summary of states populated with excitation energy and the cross section at the most forward angle in the distribution $\left(\theta_{\text {c.m. }}\right)$. The $\ell$ transfer, assumed $J^{\pi}$, and spectroscopic factors $S$ are also given. $S$ for the ground and $55-\mathrm{keV}$ states are deduced from a combined fit of angular distributions. Integrated cross sections are given for states above $5500 \mathrm{keV}$, for the two array positions, along with $S$ for $J^{\pi}=1 / 2^{-}, 3 / 2^{-}$, or $5 / 2^{-}$.

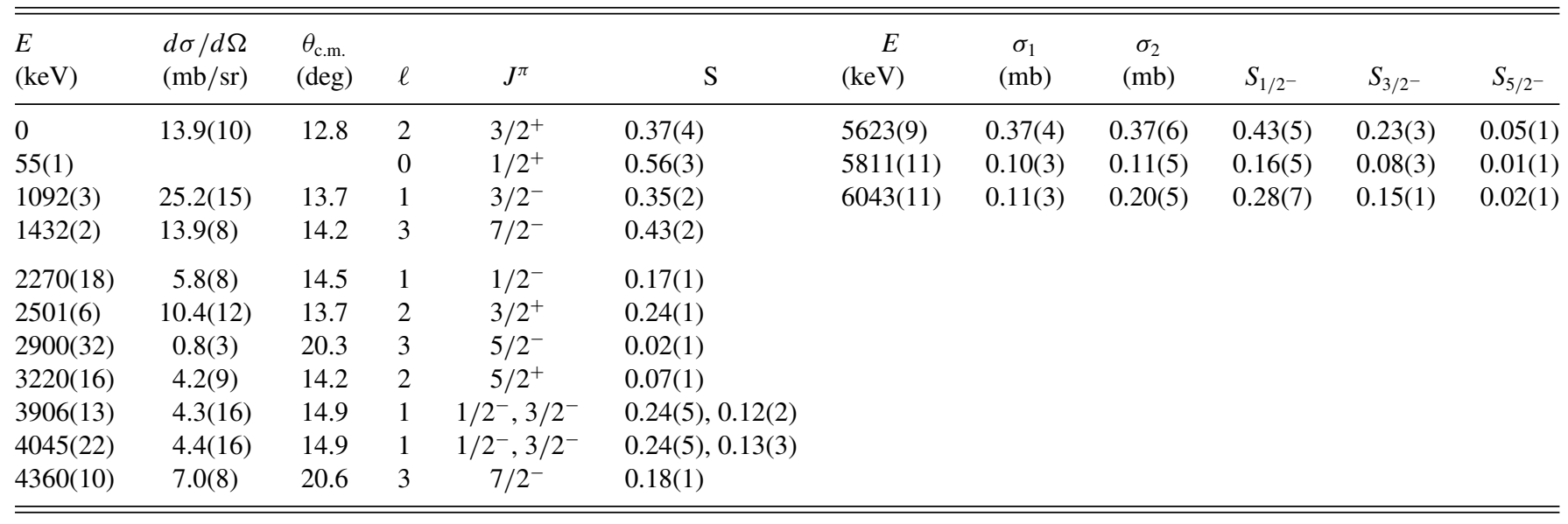

interaction was developed to better describe the behavior of negative-parity states in this region, by considering a wider range of single-particle energies (SPEs) and two-body matrix elements (TBMEs) for both $s d-f p$ and the $f p$ subspaces in the fitting procedure [38]. Both SDPF-MU and FSU interactions use $0 \mathrm{p}-0 \mathrm{~h}$ excitations for even-parity states and $1 \mathrm{p}-1 \mathrm{~h}$ for negative-parity ones. The EEdf1 interaction has been derived using chiral effective-field theory using more than one major oscillator shell, without fitting any SPEs or TBMEs. It includes three-body interactions and aims to better describe the properties of neutron-rich $\mathrm{Ne}, \mathrm{Mg}$, and $\mathrm{Si}$ isotopes.

Comparison of experimental levels with calculations can be used to suggest spin assignments. The states at 1432 and $4360 \mathrm{keV}$ with $\ell=3$ correspond well to predicted $J^{\pi}=7 / 2^{-}$ states at those energies. The third state populated via an $\ell=3$ transfer at $2900 \mathrm{keV}$ is similar in energy and strength to a predicted $5 / 2^{-}$state in the FSU and EEdf1 calculations. The $\ell=2$ ground state and $2501-\mathrm{keV}$ state are both taken as $J^{\pi}=3 / 2^{+}$; the ground state is a known $3 / 2^{+}$state [3] and the strongly populated excited $3 / 2^{+}$state compares well to the calculations. There is a weaker $\ell=2$ state at $3220 \mathrm{keV}$ that corresponds reasonably to a calculated $5 / 2^{+}$state. Similarly, the $\ell=1$ states at 1092 and $2270 \mathrm{keV}$ are assigned as $3 / 2^{-}$ and $1 / 2^{-}$states, respectively. The $\ell=1$ peak at $3980 \mathrm{keV}$ is a likely doublet, given the measured width; a single state at this energy and width would have an expected $S \approx 1$. This is inconsistent with the value of $S$ extracted on the basis of either a single $3 / 2^{-}$state $(S=0.25)$ or $1 / 2^{-}$state $(S=0.50)$, but matches the shell-model calculations, which predict a doublet of two $\ell=1$ states with $J^{\pi}=1 / 2^{-}$and $3 / 2^{-}$close to the measured energies. However, there were insufficient data to fit a doublet to spectra at each angle. Each state should have a similar angular distribution and so a ratio of yields for these states was extracted from the spectrum across the entire angular range. The spectrum in Fig. 1 shows the fit to the integrated yields while the angular distribution in Fig. 2 is for the combined yield. A summary of the observed states is given in Table I.
All the calculations reproduce the gross features of the measured single-particle strength distributions in ${ }^{29} \mathrm{Mg}$, although root-mean-squared deviations from experimental energies for the FSU and EEdf1 interactions are $\approx 300 \mathrm{keV}$ lower than for SDPF-MU.

Vacancies deduced from summed single-particle strength for the $s_{1 / 2}, p, d$, and $f_{7 / 2}$ orbitals are given in Table II for ${ }^{29} \mathrm{Mg}$, compared with ${ }^{31} \mathrm{Si}[32]$ and ${ }^{33} \mathrm{~S}[33,34]$. The vacancies for the two $p$ orbitals were combined to avoid issues with $j$ assignments for some $\ell=1$ states, but unassigned $\ell=2$ strength was taken to be $d_{3 / 2}$ and unassigned $\ell=3$ strength above $7 \mathrm{MeV}$ was assumed to be $f_{5 / 2}$. Uncertainties in centroids incorporate any ambiguities in $j$ assignments.

Strength appears to be missing for the $p$ orbitals in ${ }^{29} \mathrm{Mg}$, compared to the other isotopes. It is probable that it lies at higher excitation, but no $\ell$ assignment could be made above $5.5 \mathrm{MeV}$. The FSU calculations predict $3 / 2^{-}$and $1 / 2^{-}$states at 5722 and $6079 \mathrm{keV}$ with spectroscopic factors of $S=0.10$ and 0.05 , respectively. Assuming that these correspond to the states observed at 5623 and $5811 \mathrm{keV}$, the summed $\ell=1$ strength becomes 3.97(22); the effect of inclusion on $p_{1 / 2}$ and $p_{3 / 2}$ centroids is shown explicitly in later figures. (For

TABLE II. Vacancies deduced from summed spectroscopic factors. For ${ }^{29} \mathrm{Mg}$, this is given up to $4.36 \mathrm{MeV}$ and the effect of including the 5623- and 5811-keV states as $\ell=1$ is indicated (see text). Cross-section data for ${ }^{31} \mathrm{Si}[32]$ and ${ }^{33} \mathrm{~S}[33,34]$ were used to obtain strength using the same methodology.

\begin{tabular}{lcll}
\hline \hline Orbital & ${ }^{29} \mathrm{Mg}$ & ${ }^{31} \mathrm{Si}$ & ${ }^{33} \mathrm{~S}$ \\
\hline$s_{1 / 2}$ & $1.13(7)$ & $0.76(6)$ & $0.39(4)$ \\
$d_{3 / 2}$ & $2.45(20)$ & $2.91(24)$ & $2.99(25)$ \\
$d_{5 / 2}$ & $0.43(3)$ & $0.32(3)$ & $0.62(4)$ \\
$p$ & $2.73(17)$ & $3.98(23)$ & $4.37(11)$ \\
$f_{7 / 2}$ & $4.81(22)$ & $6.40(55)$ & $6.13(50)$ \\
Total & $11.5(4)$ & $14.4(7)$ & $14.5(6)$ \\
Total + higher states & $12.7(5)$ & & \\
\hline \hline
\end{tabular}



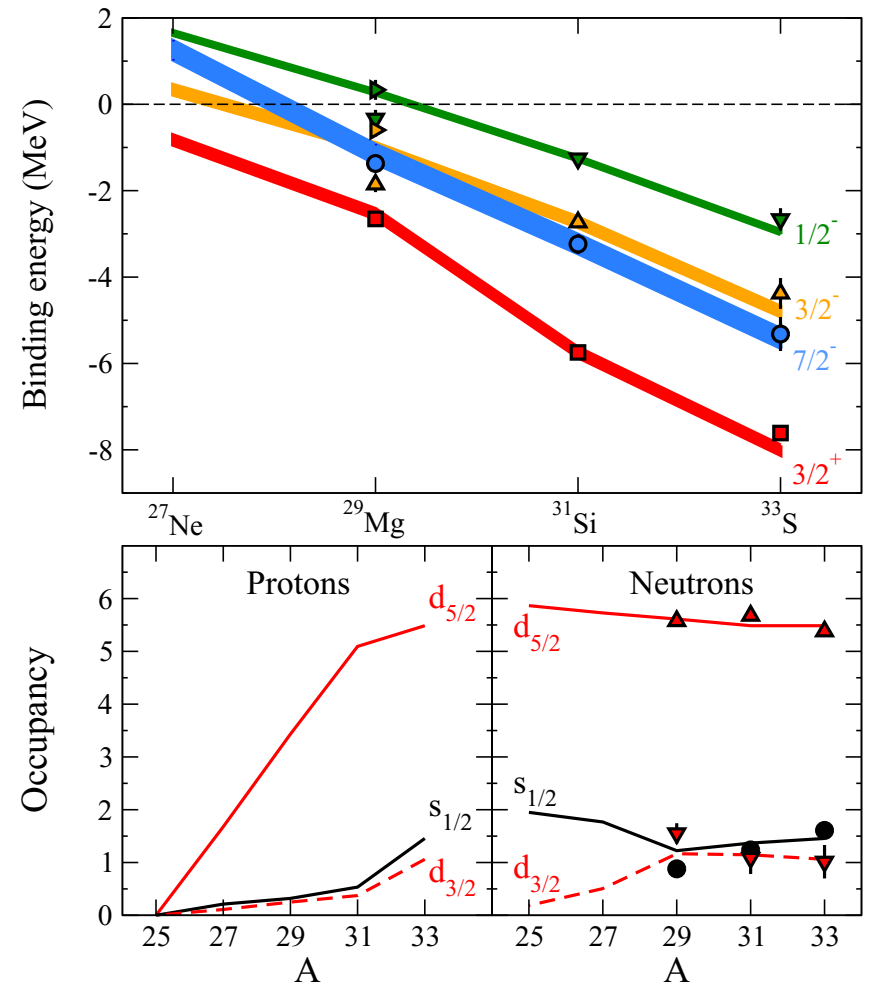

FIG. 4. (Top) Binding energy of single-particle centroids for $J^{\pi}=3 / 2^{+}(\square), 7 / 2^{-}(\bigcirc), 3 / 2^{-}(\triangle)$, and $1 / 2^{-}(\nabla)$ in $N=17$ isotones. Centroids for $J^{\pi}=1 / 2^{-}$and $3 / 2^{-}$, including states above $5.5 \mathrm{MeV}$, are denoted by $\triangleright$. Data for ${ }^{31} \mathrm{Si}$ and ${ }^{33} \mathrm{~S}$ are from Refs. [32-34]. Bands represent calculations using the FSU interaction, with widths representing experimental uncertainties in ${ }^{31} \mathrm{Si}$, onto which they are shifted. (Bottom) Calculated nucleon occupancies for $N=17$ isotones from FSU interaction (lines). Symbols represent neutron occupancies extracted from the data.

completeness, the state at $6043 \mathrm{keV}$ matches best in strength to a predicted $5 / 2^{-}$state.)

The total strength in these orbitals is then consistent across the three isotopes with an rms deviation of around $10 \%$. The associated single-particle centroids are compared to those from the FSU shell-model calculations in Fig. 4, shifted onto the experimental data for ${ }^{31} \mathrm{Si}$. There is excellent agreement between observed changes in binding energy and the calculations. The shell-model interactions seem to describe the ${ }^{29} \mathrm{Mg}$ data and the evolution of single-particle centroids away from stability reasonably well with only the requirement of $0 \mathrm{p}-0 \mathrm{~h}$ and $1 \mathrm{p}-1 \mathrm{~h}$ excitations for positive- and negative-parity states, respectively. Qualitatively the evolution can be described in terms of the interaction between $d_{5 / 2}$ protons (filling between oxygen and silicon as shown in Fig. 4) and different neutron orbitals, whose occupancies are similar for magnesium, silicon, and sulfur [39]. Indeed, the experimentally deduced neutron occupancies agree well with the calculations, as shown in Fig. 4, but data on protons are not available. The $\pi d_{5 / 2}$ orbital has the largest overlap with the $v d_{3 / 2}$ orbital, followed by the $v f_{7 / 2}$ and $v p_{3 / 2}$. As the $\pi d_{5 / 2}$ orbital fills, the attractive monopole interaction between nucleons reduces the energy of the different neutron orbitals dependent on the

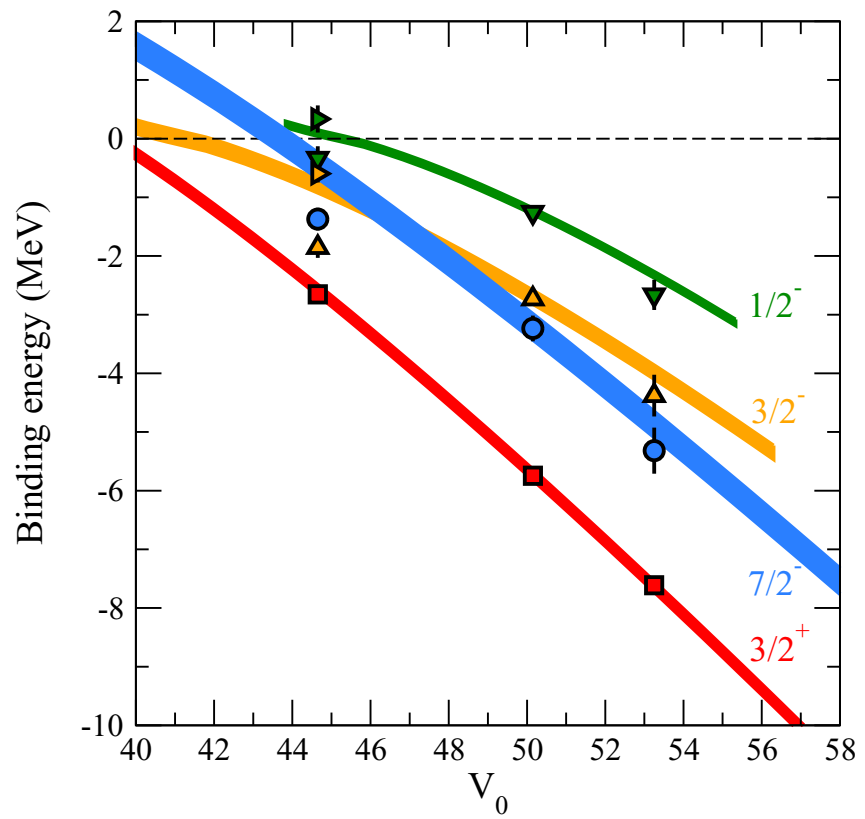

FIG. 5. Binding energies calculated using Woods-Saxon formalism with fixed geometry (see text); bands include experimental uncertainties for ${ }^{31} \mathrm{Si}$, to which the calculations have been fixed. Symbols have the same meaning as Fig. 4.

size of this overlap. Additionally, the tensor component of the neutron-proton interaction will be attractive for the $v d_{3 / 2}$ and repulsive for the $v f_{7 / 2}$ and $v p_{3 / 2}$ orbitals. The overall effect of these interactions results in the $N=20$ shell closure at stability. Removing these protons reduces the difference in energy between the $d_{3 / 2}$ and $f p$-shell neutrons and thus the magnitude of the shell closure and changes in the ordering of the $f p$ orbitals. This is known as type-I shell evolution [40]. Beyond magnesium, the emergence of the $N=16$ shell closure between the $s_{1 / 2}$ and $d_{3 / 2}$ orbitals is predicted to result in changes to neutron occupancies, which then affects the energy spacing between $d_{3 / 2}$ and $f_{7 / 2}$ neutrons in the opposite sense, though more experimental data are needed to substantiate this behavior.

Given the proximity to the neutron-separation threshold, the effect of the finite geometry of the potential could play a role. The influence of the geometry of a finite potential well can cause low- $\ell$ orbitals to linger near threshold, where the rate of change of eigenstate energies decreases as they approach zero binding, as described in Ref. [41] for $p$ orbitals. Figure 5 shows calculations using a Woods-Saxon potential with fixed geometry for $A=31\left(r=1.2 A^{1 / 3} \mathrm{fm}\right.$, $\left.r_{s o}=1.1 A^{1 / 3}, a_{0}=0.65 \mathrm{fm}\right)$. The depth of the potential $V_{0}$ is chosen to reproduce the binding energy for the $d_{3 / 2}$ orbital. The calculated trends for the excited levels were normalized to the experimental values at ${ }^{31} \mathrm{Si}$. The relative changes in binding energy are generally well reproduced, with the exception of the $f_{7 / 2}$ centroid in ${ }^{29} \mathrm{Mg}$. The behavior of both $p$-orbital centroids as they approach threshold is also reproduced, accounting for a reduction in energy between the $p_{1 / 2}$ and $p_{3 / 2}$ orbitals. The effects of a finite geometry are not explicitly accounted for in shell-model calculations. However, given the 
success of the FSU interaction in describing the observed trends, it appears that this has been captured, perhaps by fitting of the effective interaction over a broader range of binding energies for the negative-parity states [42].

In summary, we performed a measurement of the ${ }^{28} \mathrm{Mg}(d, p)$ reaction in inverse kinematics, marking an early exploitation of the ISOLDE Solenoidal Spectrometer. The excellent resolution provided by this technique allowed a comprehensive study of single-particle states populated in ${ }^{29} \mathrm{Mg}$, where the majority of states up to $4.36 \mathrm{MeV}$ have been resolved. The properties of states above the neutron separation energy have also been determined. Comparisons with calculations suggest proton-neutron interactions are driving the evolution of shell structure; however, as $p$-shell levels approach the neutron-separation energy, the effects of finitepotential geometry are important considerations, in particular, explaining the reduction in separation of the $p$ orbitals.

New interactions including cross-shell effects reproduce the distribution of single-particle strength in ${ }^{29} \mathrm{Mg}$ reasonably well. They compare well to broader trends in the $N=17$ isotones, where calculations extend to ${ }^{25} \mathrm{O}$. The precision data on the negative-parity states in ${ }^{29} \mathrm{Mg}$ will serve to improve these calculations further, while also providing a valuable benchmark just outside the island of inversion. Future experiments, such as ${ }^{30} \mathrm{Mg}(d, p){ }^{31} \mathrm{Mg}$ studies, will follow this evolution of strength into the region of inversion, where type-II shell evolution arising due to increases in particle-hole excitations [40] is likely to play an important role.

Acknowledgments. This measurement (IS621) was carried out at the ISOLDE facility at CERN. We wish to thank the team at ISOLDE for their commitment to ensuring this measurement with the ISS could take place before CERN's long shutdown. We would also like to thank the engineering support provided by Daresbury Laboratory. This work was supported by the U.K. Science and Technology Facilities Council [Grants No. ST/P004598/1, No. ST/N002563/1, No. ST/M00161X/1 (Liverpool), No. ST/P004423/1 (Manchester), No. ST/P005314/1 (Surrey), the ISOL-SRS Grant (Daresbury), No. ST/R004056/1 (Ernest Rutherford Fellowship - Gaffney), and No. ST/T004797/1 (Ernest Rutherford Fellowship - Sharp)], the U.S. Department of Energy, Office of Science, Office of Nuclear Physics, under Contracts No. DE-AC02-06CH11357 (ANL) and No. DE-SC-0014552 (UConn), the European Union's Horizon 2020 Framework research and innovation program under Grant Agreement No. 654002 (ENSAR2), the Marie Skłodowska-Curie Grant Agreement No. 665779, the Research Foundation Flanders (FWO, Belgium), the European Research Council under the European Union's Seventh Framework Programme (FP7/2007-2013)/ERC Grant Agreement No. 617156, and the Spanish Ministry of Science and Innovation under Grants No. PGC2018-095640B-I00"ELEGANT" and No. PID2019-104390GB-I00. This research used targets provided by the Center for Accelerator Target Science at Argonne National Laboratory. The FSU shell-model calculations were performed using the computational facility of the nuclear physics theory group, Florida State University, supported by grants from the U.S. Department of Energy, Office of Science (DE-SC-0009883 (FSU).
[1] C. Thibault, R. Klapisch, C. Rigaud, A. M. Poskanzer, R. Prieels, L. Lessard, and W. Reisdorf, Phys. Rev. C 12, 644 (1975).

[2] E. K. Warburton, J. A. Becker, and B. A. Brown, Phys. Rev. C 41, 1147 (1990).

[3] M. Kowalska, D. T. Yordanov, K. Blaum, P. Himpe, P. Lievens, S. Mallion, R. Neugart, G. Neyens, and N. Vermeulen, Phys. Rev. C 77, 034307 (2008).

[4] N. Imai, M. Mukai, J. Cederkäll, H. Aghai, P. Golubev, H. T. Johansson, D. Kahl, J. Kurcewics, T. Teranishi, and Y. X. Watanabe, Phys. Rev. C 90, 011302(R) (2014).

[5] R. V. F. Janssens, Nature (London) 459, 1069 (2009).

[6] E. K. Warburton and B. A. Brown, Phys. Rev. C 46, 923 (1992).

[7] P. C. Bender, C. R. Hoffman, M. Wiedeking, J. M. Allmond, L. A. Bernstein, J. T. Burke, D. L. Bleuel, R. M. Clark, P. Fallon, B. L. Goldblum, T. A. Hinners, H. B. Jeppesen, Sangjin Lee, I.-Y. Lee, S. R. Lesher, A. O. Macchiavelli, M. A. McMahan, D. Morris, M. Perry, L. Phair, et al. Phys. Rev. C 80, 014302 (2009).

[8] D. Steppenbeck, A. N. Deacon, S. J. Freeman, R. V. F. Janssens, M. P. Carpenter, C. R. Hoffman, B. P. Kay, T. Lauritsen, C. J. Lister, D. O’Donnell, J. Ollierd, D. Seweryniak, J. F. Smith, K.-M. Spohr, S. L. Tabor, V. Tripathi, P. T. Wady, and S. Zhu, Nucl. Phys. A. 847, 149 (2010).
[9] Y. Utsuno, T. Otsuka, T. Mizusaki, and M. Honma, Phys. Rev. C 60, 054315 (1999).

[10] S. M. Brown, W. N. Catford, J. S. Thomas, B. FernándezDomínguez, N. A. Orr, M. Labiche, M. Rejmund, N. L. Achouri, H. Al Falou, N. I. Ashwood, D. Beaumel, Y. Blumenfeld, B. A. Brown, R. Chapman, M. Chartier, N. Curtis, G. de France, N. de Sereville, F. Delaunay, A. Drouart, C. Force, S. Franchoo, et al. Phys. Rev. C 85, 011302(R) (2012).

[11] P. Baumann, P. Dessagne, A. Huck, G. Klotz, A. Knipper, G. Marguier, C. Miehé, M. Ramdane, C. Richard-Serre, G. Walter, and B. H. Wildenthal, Phys. Rev. C 36, 765 (1987).

[12] T. Shimoda, K. Tajiri, K. Kura, A. Odahara, M. Suga, Y. Hirayama, N. Imai, H. Miyatake, M. Pearson, C. D. P. Levy, K. P. Jackson, R. Legillon, C. Petrache, T. Fukuchi, N. Hamatani, T. Hori, M. Kazato, Y. Kenmoku, T. Masue, H. Nishibata, et al. Hyperfine Interact. 225, 183 (2014).

[13] D. K. Scott, B. G. Harvey, D. L. Hendrie, L. Kraus, C. F. Maguire, J. Mahoney, Y. Terrien, and K. Yagi, Phys. Rev. Lett 33, 1343 (1974).

[14] L. K Fifield, P. V. Drumm, M. A. C. Hotchkis, T. R. Ophel, and C. L. Woods, Nucl. Phys. A 437, 141 (1985).

[15] C. L. Woods, W. N. Catford, L. K. Fifield, N. A. Orr, and R. J. Sadleir, Nucl. Phys. A 476, 392 (1988).

[16] J. R. Terry, B. A. Brown, C. M. Campbell, J. M. Cook, A. D. Davies, D.-C. Dinca, A. Gade, T. Glasmacher, P. G. Hansen, 
B. M. Sherrill, H. Zwahlen, D. Bazin, K. Yoneda, J. A. Tostevin, T. Otsuka, Y. Utsuno, and B. Pritychenko, Phys. Rev. C 77, 014316 (2008).

[17] A. Matta, W. N. Catford, N. A. Orr, J. Henderson, P. Ruotsalainen, G. Hackman, A. B. Garnsworthy, F. Delaunay, R. Wilkinson, G. Lotay, Naofumi Tsunoda, Takaharu Otsuka, A. J. Knapton, G. C. Ball, N. Bernier, C. Burbadge, A. Chester, D. S. Cross, S. Cruz, C. Aa. Diget, T. Domingo, et al. Phys. Rev. C 99, 044320 (2019).

[18] A. H. Wuosmaa, J. P. Schiffer, B. B. Back, C. J. Lister, and K. E. Rehm, Nucl. Instrum. Meth. A 580, 1290 (2007).

[19] J. C. Lighthall, B. B. Back, S. I. Baker, S. J. Freeman, H. Y. Lee, B. P. Kay, S. T. Marley, K. E. Rehm, J. E. Rohrer, J. P. Schiffer, D. V. Shetty, A. W. Vann, J. R. Winkelbauer, and A. H. Wuosmaa, Nucl. Instrum. Meth. A 622, 97 (2010).

[20] Y. Kadi, Y. Blumenfeld, W. Venturini Delsolaro, M. A. Fraser, M. Huyse, A Papageorgiou Koufidou, J. A. Rodriguez, and F. Wenander, J. Phys. G: Nucl. Part. Phys. 44, 084003 (2017).

[21] V. Fedosseev, K. Chrysalidis, T. Day Goodacre, B. Marsh, S. Rothe, C. Seiffert, and K. Wendt, J. Phys. G: Nucl. Part. Phys. 44, 084006 (2017).

[22] F. Ames, G. Bollen, P. Delahaye, O. Forstner, G. Huber, O. Kester, K. Reisinger, and P. Schmidt, Nucl. Instrum. Meth. A 538, 17 (2005).

[23] F. Wenander, JINST 5, C10004 (2010).

[24] P. T. MacGregor, Ph.D. thesis, University of Manchester, Manchester, UK, 2021 (unpublished).

[25] M. Shamsuzzoha Basunia, Nucl. Data Sheets 113, 909 (2012).

[26] P. D. Kunz, Computer code DWUCK5 (unpublished).

[27] B. P. Kay, J. P. Schiffer, and S. J. Freeman, Phys. Rev. Lett. 111, 042502 (2013).
[28] C. M. Vincent and H. T. Fortune, Phys. Rev. C 2, 782 (1970).

[29] M. Wang, G. Audi, A, H. Wapstra, F. G. Kondev, M. MacCormick, X. Xu, and B. Pfeiffer, Chin. Phys. C 36, 1603 (2012).

[30] Please see the Supplemental Material at http://link.aps. org/supplemental/10.1103/PhysRevC.104.L051301 for detailed data on state-by-state cross sections.

[31] M. H. Macfarlane and J. B. French, Rev. Mod. Phys. 32, 567 (1960).

[32] Š. Piskoř, J. Novák, E. Šimečková, J. Cejpek, V. Kroha, J. Dobeš, P. Navrátil, Nucl. Phys. A 662, 112 (2000).

[33] M. C. Mermaz, C. A. Whitten, Jr., J. W. Champlin, A. J. Howard, and D. A. Bromley, Phys. Rev. C 4, 1778 (1971).

[34] R. Liljestrand, J. McIntyre, G. Blanpied, J. Lynch, L. Ray, W. R. Coker, and G. W. Hoffmanny, Phys. Rev. C 11, 1570 (1975).

[35] Y. Utsuno (private communication).

[36] R. S. Lubna (private communication).

[37] N. Tsunoda, T. Otsuka, N. Shimizu, M. Hjorth-Jensen, K. Takayanagi, and T. Suzuki, Phys. Rev. C. 95, 021304(R) (2017).

[38] R. S. Lubna, K. Kravvaris, S. L. Tabor, Vandana Tripathi, A. Volya, E. Rubino, J. M. Allmond, B. Abromeit, L. T. Baby, and T. C. Hensley, Phys. Rev. C. 100, 034308 (2019).

[39] O. Sorlin and M.-G. Porquet, Prog. Part. Nucl. Phys. 61, 602 (2008).

[40] T. Otsuka and Y. Tsunoda, J. Phys. G: Nucl. Part. Phys. 43, 024009 (2016).

[41] B. P. Kay, C. R. Hoffman, and A. O. Macchiavelli, Phys. Rev. Lett. 119, 182502 (2017).

[42] R. S. Lubna, K. Kravvaris, S. L. Tabor, Vandana Tripathi, E. Rubino, and A. Volya, Phys. Rev. Res. 2, 043342 (2020). 